\title{
Dual-Functional Terahertz Waveplate Based on All-Dielectric Metamaterial
}

\author{
Jianchen Zi, ${ }^{1}$ Yanfeng Li®, ${ }^{1, *}$ Xi Feng, ${ }^{1,2}$ Quan Xu,${ }^{1}$ Hongchao Liu, ${ }^{3}$ Xi-Xiang Zhang,,${ }^{2,}$ \\ Jiaguang Han, ${ }^{1, \$}$ and Weili Zhang ${ }^{1,4}$ \\ ${ }^{1}$ Center for Terahertz Waves and College of Precision Instrument and Optoelectronics Engineering, and \\ Key Laboratory of Optoelectronic Information Technology (Ministry of Education), Tianjin University, \\ Tianjin 300072, China \\ ${ }^{2}$ Division of Physical Science and Engineering, King Abdullah University of Science and Technology, \\ Thuwal 23955-6900, Saudi Arabia \\ ${ }^{3}$ Joint Key Laboratory of the Ministry of Education, Institute of Applied Physics and Materials Engineering, \\ University of Macau, Avenida da Universidade, Taipa, Macau SAR, China \\ ${ }^{4}$ School of Electrical and Computer Engineering, Oklahoma State University, Stillwater, Oklahoma 74078, USA
}

(Received 11 December 2019; revised manuscript received 14 January 2020; accepted 27 February 2020; published 17 March 2020)

Terahertz technology has attracted wide attention because of its potential applications in diverse fields, such as spectroscopy, imaging, and nondestructive evaluation, but the lack of functional devices, including polarization convertors, is still a major hurdle for these applications. In previous studies on metamaterialbased polarization convertors, only one single polarization conversion is generally achieved. This paper presents a transmissive dual-functional terahertz waveplate based on an all-dielectric metamaterial composed of periodic subwavelength pillars made of pure silicon that can achieve two different polarization conversions (i.e., a combination of quarter- and half-waveplates) at the same operation frequency and for $x$ - and $y$-polarized incidences, respectively. Theoretical and numerical analyses are provided to illustrate the design principle. A sample is fabricated and characterized, and the experimental results show that the two polarization conversions are obtained at $1.01 \mathrm{THz}$, which is in good agreement with the corresponding simulations. Such a dual-functional terahertz waveplate will find applications in terahertz systems and the design strategy can be extended to other frequency ranges as well.

DOI: 10.1103/PhysRevApplied.13.034042

\section{INTRODUCTION}

Terahertz (THz) waves usually refer to waves falling within the electromagnetic frequency band of $0.1-10 \mathrm{THz}$. Due to their unique properties, $\mathrm{THz}$ waves find important applications in such fields as spectroscopy [1], imaging [2], and communications [3] and have attracted great research interest over the past decade. However, the diverse applications of $\mathrm{THz}$ technology call for the development of efficient sources, detectors, and high-performance functional devices, such as absorbers [4-6], filters [7], sensors [8], modulators [9,10], polarizers [11], splitters [12], and polarization convertors [13], many of which are achieved with metamaterials.

Waveplates, as a basic polarization convertor, are widely applied in $\mathrm{THz}$ systems. Traditional waveplates based on material birefringence [14-16] have the disadvantage of

\footnotetext{
*yanfengli@tju.edu.cn

†xixiang.zhang@kaust.edu.sa

†jiaghan@tju.edu.cn
}

being bulky due to the low birefringence of natural materials, while metamaterials and particularly metasurfaces can greatly reduce their thickness and provide a route towards more compact and potentially integrated planar devices.

Metamaterials, which are artificial materials composed of subwavelength elements [17-21], can be flexibly designed to achieve various unusual functions that cannot be fulfilled with traditional materials and are often used to design functional devices required for $\mathrm{THz}$ waves. Many $\mathrm{THz}$ polarization convertors based on metamaterials are designed to replace traditional waveplates [2232]. Grady et al. demonstrated metamaterial-based $\mathrm{THz}$ polarization converters capable of rotating a linear polarization state into its orthogonal one [22]. Yang et al. achieved linear cross-polarization conversion with a metasurface reflectarray composed of high-refractive-index silicon wires and a silver ground plane [23]. Liu et al. proposed single-layer plasmonic metasurface half-waveplates (HWPs) with a wavelength-independent polarization conversion angle [25]. Ke et al. presented a $\mathrm{THz}$ quarterwaveplate (QWP) composed of double-stacked hyperbolic metamaterial waveguide arrays [26]. Woo et al. reported a 
cross-polarization conversion by a through-via connected double-layer slot structure working in the $\mathrm{THz}$ frequency range [27]. However, these passive convertors usually possess only one function at the operation frequency, whereas the freedom of design and flexibility enabled by metamaterials allow the fabrication of versatile polarization convertors, which is highly attractive for device applications with respect to miniaturization and integration.

Here, we present a transmissive dual-functional $\mathrm{THz}$ waveplate based on an all-dielectric metamaterial (ADMM) composed of periodic unit cells fabricated on a silicon wafer. Unlike the ADMM devices previously synthesized that use a single-polarization conversion, such as a QWP or HWP, at the chosen frequency [33,34], the waveplate reported here can achieve two different polarization conversions of linear-to-circular polarization and linearto-cross-polarization at the same frequency for $x$ - and $y$-polarized incidences, respectively. Numerical simulations and experimental results are in very good agreement, which validates our design strategy. The dual-functional terahertz waveplate is expected to be of great value and used in THz systems.

\section{DESIGN STRATEGY}

In this section, we present our design strategy of the ADMM device using theoretical and numerical analyses. Theoretical analyses for polarization conversion are typically done with the help of the Jones vectors [35]. In $x, y$ coordinates, the electric field is expressed as a $2 \mathrm{D}$ vector

$$
E=\left[\begin{array}{c}
E_{x} \\
E_{y}
\end{array}\right]
$$

where $E_{x} \quad\left(E_{y}\right)$ corresponds to the $x$-polarized $(y$-polarized) component. For example, $x$-polarized, $y$-polarized, and left-circular polarizations (LCPs) can be simply expressed as

$$
\begin{aligned}
E_{x-\mathrm{pol}} & =\left[\begin{array}{l}
1 \\
0
\end{array}\right], \\
E_{y-\mathrm{pol}} & =\left[\begin{array}{l}
0 \\
1
\end{array}\right], \\
E_{\mathrm{LCP}} & =\left[\begin{array}{c}
1 \\
-i
\end{array}\right] .
\end{aligned}
$$

The transmission matrix $T$ of a polarization device can be denoted by

$$
T=\left[\begin{array}{ll}
T_{x x} & T_{x y} \\
T_{y x} & T_{y y}
\end{array}\right]
$$

where $T_{x x}\left(T_{y y}\right)$ is the complex transmission coefficient for copolarization, and $T_{x y}\left(T_{y x}\right)$ is for cross-polarization.
We assume that the ADMM device can convert an $x$ polarized incidence into an LCP output at the operation frequency of $1 \mathrm{THz}$ and convert a $y$-polarized incidence into an $x$ polarization at the same frequency. We then obtain

$$
\begin{aligned}
k_{1} E_{\mathrm{LCP}} & =T \cdot E_{x-\mathrm{pol}}, \\
k_{2} E_{x-\mathrm{pol}} & =T \cdot E_{y-\mathrm{pol}},
\end{aligned}
$$

where $k_{1}$ and $k_{2}$ are arbitrary nonzero complex numbers corresponding to an overall conversion efficiency and phase that have no effect on the polarization state. We substitute Eqs. (2a)-(2c) and (3) into Eqs. (4a) and (4b) and obtain

$$
\begin{gathered}
T_{y x}=-i T_{x x} \neq 0, \\
T_{x y} \neq 0, \\
T_{y y}=0 .
\end{gathered}
$$

That is, to realize the expected dual-functional performance, the transmission matrix $T$ of the ADMM needs to satisfy the above conditions.

The transmission matrix $T$ is determined by the unit cell of the ADMM. From our previous work [33,34], we know that an ADMM with a single subwavelength anisotropic pillar (hole) as the unit cell works in the effective medium regime [36] and that it can result in birefringence. According to symmetry, the main axes of the unit cell are along the two symmetric axes of the pillar (hole). These two main axes can be denoted as the $a$ and $b$ directions. The incidences polarized along the $a$ and $b$ axes will have different phase delays and no cross-polarized output. The transmis-

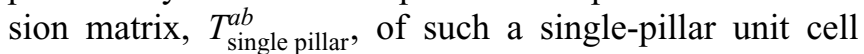
(SPUC) in $a, b$ coordinates can be represented as

$$
T_{\text {single pillar }}^{a b}=\left[\begin{array}{cc}
t_{a} e^{i \delta_{a}} & 0 \\
0 & t_{b} e^{i \delta_{b}}
\end{array}\right],
$$

where $t_{a}\left(t_{b}\right)$ and $\delta_{a}\left(\delta_{b}\right)$ correspond to the transmission amplitude and phase for $a$-polarized ( $b$-polarized) incidence, respectively. Moreover, the difference in transmission amplitudes is usually small, and it can be assumed that $t_{a}=t_{b}=t$, which is nonzero. If the $a, b$ coordinates have an in-plane anticlockwise rotation angle $\theta$ with respect to the $x, y$ coordinates, the transmission matrix in the $x, y$ coordinates can be obtained by performing matrix rotations in Eq. (6),

$$
\begin{aligned}
T_{\text {single pillar }}^{x y} & {\left[\begin{array}{cc}
\cos \theta & -\sin \theta \\
\sin \theta & \cos \theta
\end{array}\right]\left[\begin{array}{cc}
t_{a} e^{i \delta_{a}} & 0 \\
0 & t_{b} e^{i \delta_{b}}
\end{array}\right] } \\
& \times\left[\begin{array}{cc}
\cos \theta & \sin \theta \\
-\sin \theta & \cos \theta
\end{array}\right] \\
= & {\left[\begin{array}{cc}
e^{i \delta_{a}} \cos ^{2} \theta+e^{i \delta_{b}} \sin ^{2} \theta & \left(e^{i \delta_{a}}-e^{i \delta_{b}}\right) \sin \theta \cos \theta \\
\left(e^{i \delta_{a}}-e^{i \delta_{b}}\right) \sin \theta \cos \theta & e^{i \delta_{a}} \sin ^{2} \theta+e^{i \delta_{b}} \cos ^{2} \theta
\end{array}\right] t . }
\end{aligned}
$$


We can see that the cross-polarized transmission coefficients $T_{x y}$ and $T_{y x}$ are identical for $T_{\text {single pillar }}^{x y}$. According to Eqs. (5a)-(5c), if a SPUC-based ADMM can realize the desired performance, it has to satisfy

$$
\begin{gathered}
t\left(e^{i \delta_{a}}-e^{i \delta_{b}}\right) \sin \theta \cos \theta=-i t\left(e^{i \delta_{a}} \cos ^{2} \theta+e^{i \delta_{b}} \sin ^{2} \theta\right) \neq 0, \\
t\left(e^{i \delta_{a}} \sin ^{2} \theta+e^{i \delta_{b}} \cos ^{2} \theta\right)=0 .
\end{gathered}
$$

Because $t$ is nonzero here, Eq. (8b) can be transformed into

$$
e^{i\left(\delta_{a}-\delta_{b}\right)} \sin ^{2} \theta+\cos ^{2} \theta=0 .
$$

Obviously, Eq. (9) can be satisfied only with $\delta_{a}-\delta_{b}=$ $\pm 180^{\circ}$ and $\theta= \pm 45^{\circ}$ or $\pm 135^{\circ}$. However, these conditions cannot satisfy Eq. (8a). Thus, it can be concluded that a SPUC-based ADMM cannot achieve the dual-functional performance.

Evidently, there are not enough degrees of design freedom to perform a dual-functional polarization conversion with the single-structure unit cell (SSUC), which has only one kind of subwavelength structure. Multistructure unit cells (MSUCs), containing several subwavelength structures, will enable higher degrees of design freedom to solve this problem; several MSUC-based metamaterials have been reported to fulfil different functions [37-42]. Yang et al. used an ADMM based on dual-pillar unit cells (DPUCs) with a phase gradient to obtain a polarization rotation and a separation of linearly polarized incident waves [37]. Yu et al. demonstrated a metasurface QWP composed of an array of plasmonic antennas with spatially varying phase and polarization responses [38]. Ding et al. achieved a HWP with a supercell-based plasmonic metasurface [39]. Deng et al. demonstrated the concept of vectorial holography based on a diatomic metasurface consisting of metamolecules formed by two orthogonal meta-atoms [40].

To achieve our goal, a DPUC similar to that in Ref. [37] is used instead. As shown in Fig. 1(a), each unit cell of the ADMM consists of two parts, both made of pure silicon only and thus forming an all-dielectric metamaterial. The bottom part is the substrate, which has no effect on the polarization conversion process. The top part is composed of periodic subwavelength pillars, which can be named the metamaterial layer (MML). The MML can convert the polarization of the $\mathrm{THz}$ wave propagating along the $z$ direction. As schematically shown in Fig. 1(b), the DPUC of the MML has two subcells, i.e., pillars $A$ and $B$. The periods of the DPUC are $P$ along the $x$ axis and $2 P$ along the $y$ axis. The pillar height is $h$, and the substrate thickness is $H$. For pillar $A$, the length and width are $a_{1}$ and $b_{1}$, respectively, and the $a_{1}$ side is at an angle of $\theta_{1}$ relative to the $x$ axis. For pillar $B$, the corresponding parameters are $a_{2}, b_{2}$, and $\theta_{2}$.

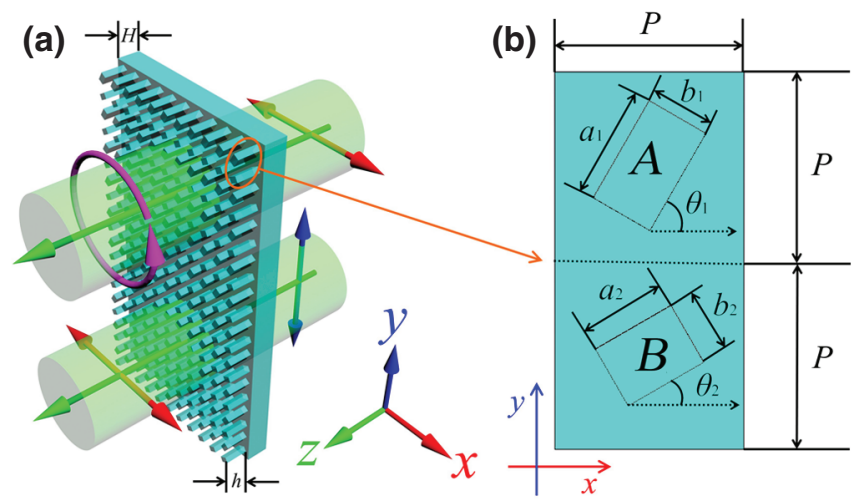

FIG. 1. (a) Schematic of the ADMM. (b) DPUC of the MML. Parameters are indicated in the figure.

Similar to Eq. (7), the transmission matrix $T_{A}\left(T_{B}\right)$ of subcell $A(B)$ in the $x, y$ coordinates can be represented as

$$
\begin{aligned}
T_{A}= & {\left[\begin{array}{cc}
\cos \theta_{1} & -\sin \theta_{1} \\
\sin \theta_{1} & \cos \theta_{1}
\end{array}\right]\left[\begin{array}{cc}
t_{1} e^{i \delta_{1}} & 0 \\
0 & t_{2} e^{i \delta_{2}}
\end{array}\right] } \\
& \times\left[\begin{array}{cc}
\cos \theta_{1} & \sin \theta_{1} \\
-\sin \theta_{1} & \cos \theta_{1}
\end{array}\right] \\
= & {\left[\begin{array}{cc}
e^{i \delta_{1}} \cos ^{2} \theta_{1}+e^{i \delta_{2}} \sin ^{2} \theta_{1} & \left(e^{i \delta_{1}}-e^{i \delta_{2}}\right) \sin \theta_{1} \cos \theta_{1} \\
\left(e^{i \delta_{1}}-e^{i \delta_{2}}\right) \sin \theta_{1} \cos \theta_{1} & e^{i \delta_{1}} \sin ^{2} \theta_{1}+e^{i \delta_{2}} \cos ^{2} \theta_{1}
\end{array}\right] t, } \\
T_{B}= & {\left[\begin{array}{cc}
\cos \theta_{2} & -\sin \theta_{2} \\
\sin \theta_{2} & \cos \theta_{2}
\end{array}\right]\left[\begin{array}{cc}
t_{3} e^{i \delta_{3}} & 0 \\
0 & t_{4} e^{i \delta_{4}}
\end{array}\right] } \\
& \times\left[\begin{array}{cc}
\cos \theta_{2} & \sin \theta_{2} \\
-\sin \theta_{2} & \cos \theta_{2}
\end{array}\right] \\
= & {\left[\begin{array}{cc}
e^{i \delta_{3}} \cos ^{2} \theta_{2}+e^{i \delta_{4}} \sin ^{2} \theta_{2} & \left(e^{i \delta_{3}}-e^{i \delta_{4}}\right) \sin \theta_{2} \cos \theta_{2} \\
\left(e^{i \delta_{3}}-e^{i \delta_{4}}\right) \sin \theta_{2} \cos \theta_{2} & e^{i \delta_{3}} \sin ^{2} \theta_{2}+e^{i \delta_{4}} \cos ^{2} \theta_{2}
\end{array}\right] t, }
\end{aligned}
$$

where $t_{1}\left(t_{2}\right)$ and $\delta_{1}\left(\delta_{2}\right)$ are, respectively, the transmission amplitude and phase for $a_{1}$-polarized ( $b_{1}$-polarized) incidence on pillar $A$, and $t_{3}\left(t_{4}\right)$ and $\delta_{3}\left(\delta_{4}\right)$ are, respectively, the transmission amplitude and phase for $a_{2}$-polarized $\left(b_{2}\right.$ polarized) incidence on pillar $B$. Again, the sizes of pillars $A$ and $B$ are very close to each other, so we assume that $t_{1}=t_{2}=t_{3}=t_{4}=t$ is satisfied. Thus, the total transmission matrix of the DPUC can be represented as

$$
T_{\text {dual pillar }}=T_{A}+T_{B} \text {. }
$$

As previously stated, $T_{\text {dualpillar }}$ should satisfy Eqs. (5a)(5c) to achieve a dual-functional polarization conversion. We then obtain four real equations with six variables $\left[\theta_{1}\right.$, $\left.\theta_{2}, \delta_{1}, \delta_{2}, \delta_{3}, \delta_{4}\right]$, and because there are more variables than equations, there is theoretically an infinite number of solutions for $\left[\theta_{1}, \theta_{2}, \delta_{1}, \delta_{2}, \delta_{3}, \delta_{4}\right]$. There is not much constraint on $\left[\theta_{1}, \theta_{2}\right]$, except some special cases, 
such as $\theta_{1}\left(\theta_{2}\right)$ being equal to $0^{\circ}, \pm 90^{\circ}$, or $\pm 180^{\circ}$. For easy fabrication and simplification of equations, $\left[\theta_{1}, \theta_{2}\right]$ will be fixed to $\left[60^{\circ}, 30^{\circ}\right]$. Finally, the equations can be solved to yield $\left[\delta_{1}, \delta_{2}, \delta_{3}, \delta_{4}\right]=\left[176^{\circ},-51^{\circ},-103^{\circ}, 31^{\circ}\right]$ within the $\left(-180^{\circ}, 180^{\circ}\right)$ range. However, it should be noted that the key to solutions is the relative phase relationship, not the absolute values. More details on how to solve the equations are provided in Section 1 within the Supplemental Material [43].

The set of values $\left[\delta_{1}, \delta_{2}, \delta_{3}, \delta_{4}\right]$ depends on the structural parameters of pillars $A$ and $B$. To get the structural parameters corresponding to the required values, as determined above, the amplitude and phase of the transmission from a silicon pillar is obtained via simulation, using the commercial software CST Microwave Studio. A silicon pillar is simulated with periodic boundary conditions in both $x$ and $y$ directions (corresponding, respectively, to $a$ and $b$ coordinates for an unrotated pillar), at an operation frequency of $1 \mathrm{THz}$, and the relative dielectric constant of silicon is set as 11.9. The period $P$, pillar height $h$, and substrate thickness $H$ are set at 150,200, and $1000 \mu \mathrm{m}$, respectively. A linear $x$-polarized plane-wave propagating along the $z$ direction is used as the incidence in the time-domain solver. Figures 2(a) and 2(b) show the distributions of the normalized transmission amplitude $t$ and phase $\delta$ as a function of the side lengths $a$ and $b$. The star markers show the best structural parameters to be used for device fabrication. From Fig. 2 , we obtain $\left[\delta_{1}, \delta_{2}, \delta_{3}, \delta_{4}\right]=\left[54^{\circ},-168^{\circ}, 138^{\circ}\right.$, $\left.-87^{\circ}\right]$, when $a_{1}=81 \mu \mathrm{m}, b_{1}=53 \mu \mathrm{m}, a_{2}=69 \mu \mathrm{m}$, and $b_{2}=48 \mu \mathrm{m}$. As mentioned above, it is the relative phase values that matter in the polarization conversion, and this set differs from the previous numerical one by about $120^{\circ}$. Additionally, $t_{1}, t_{2}, t_{3}$, and $t_{4}$ are very close to each other, and therefore, can be assumed to be equal, which justifies our previous approximation.

A discussion and justification of the working principle of our design is in order. Our structure is periodic and, using the grating equation, one can show that there is no high-order diffraction from it. Therefore, there is only the zeroth transmission order and the wave front experiences a periodic modulation. In addition, our structure with subwavelength subunits does not depend on Mie resonances, and therefore, falls into the effective medium type [36], where the $\mathrm{THz}$ wave will experience an effective index of refraction. Each silicon pillar behaves like an individual elliptical waveguide, as described in Refs. [44-46], and a difference in the effective refractive indices of the eigenmodes leads to an accumulated phase difference. The simulated eigenmodes of a unit cell are shown in Fig. S1 within the Supplemental Material [43], where it can be seen that there is a very weak interaction between subcells, justifying our use of Eq. (11) as the transmission matrix for the dual pillar. More importantly, it is also proved in the Supplemental Material [43] that the dual-pillar structure is not a simple combination of a QWP and HWP. Although pillars $A$ and $B$ act as individual waveguides, it is their interference as a whole that gives the overall polarization response.

\section{RESULTS AND DISCUSSION}

To verify the dual functions of the ADMM device, a three-dimensional (3D) model with structural parameters $a_{1}=81 \mu \mathrm{m}, b_{1}=53 \mu \mathrm{m}, a_{2}=69 \mu \mathrm{m}, b_{2}=48 \mu \mathrm{m}$, $h=200 \mu \mathrm{m}, H=1000 \mu \mathrm{m}, P=150 \mu \mathrm{m}, \theta_{1}=60^{\circ}$, and $\theta_{2}=30^{\circ}$ is built for simulation. Due to the periodic nature of the ADMM, the 3D model has only one unit cell, with periodic boundary conditions set in the $x$ and $y$ directions. Linear $x$ - and $y$-polarized THz plane waves propagating along the $z$ direction are used as incidences in the timedomain solver, respectively. Moreover, an air reference is used for normalization. Transmission amplitude $t_{i j}$, phase difference $\Delta \delta_{i}$, and ellipticity $E P_{i}$ are used to characterize the polarization properties of the ADMM, and they are defined by

$$
t_{i j}(f)=\frac{E_{i j}(f)}{E_{R}(f)},
$$
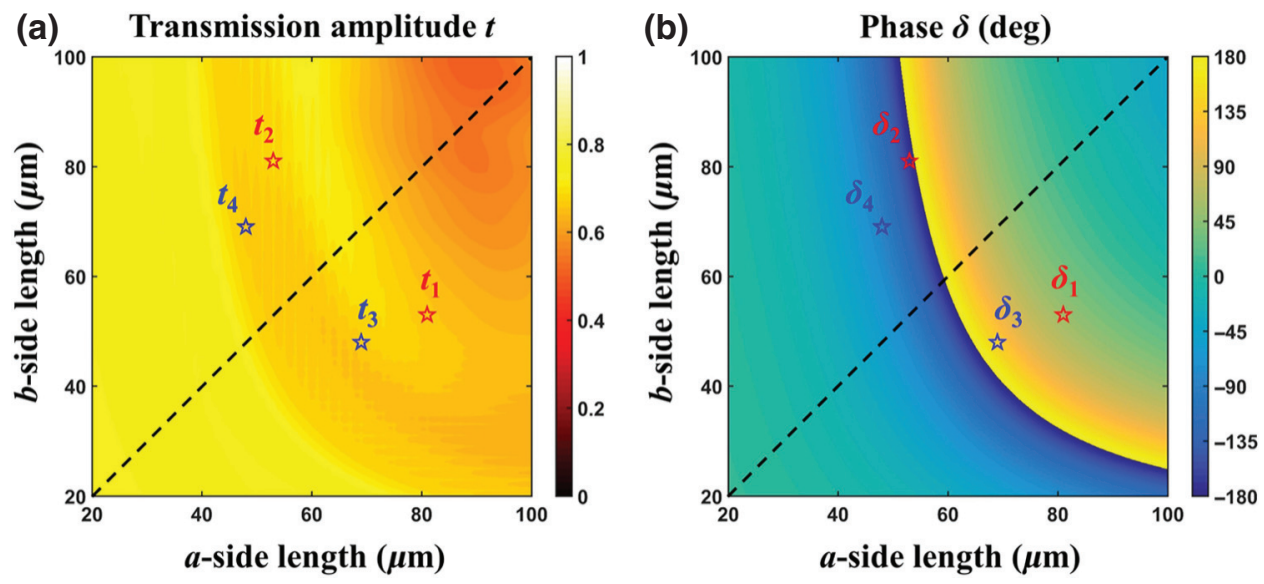

FIG. 2. (a) Simulated transmission amplitude $t$ and (b) phase $\delta$ from a silicon pillar for $\mathrm{THz}$ wave at $1 \mathrm{THz}$. 


$$
\begin{aligned}
\Delta \delta_{i}(f) & =\delta_{i y}(f)-\delta_{i x}(f), \\
E P_{i}(f) & =\frac{2 E_{i x} E_{i y} \sin \Delta \delta_{i}}{E_{i x}{ }^{2}+E_{i y}{ }^{2}},
\end{aligned}
$$

where $f$ is the frequency of the THz wave, $E_{i j}(f)$ is the amplitude of the transmitted electric field through the ADMM, and subscript $i(j)$ represents the linear polarization direction of incidence (detection). For example, subscript $x y$ means $x$-polarized incidence and $y$-polarized detection. $E_{R}(f)$ is the electric field amplitude of the reference, and $\delta_{i x}\left(\delta_{i y}\right)$ is the $x$-polarized ( $y$-polarized) phase of the transmitted electric field through the ADMM.

Simulated results are shown in Figs. 3(a)-3(c). We can see that $t_{x x}, t_{x y}$, and $t_{y x}$ are equal to approximately 0.36 at $1 \mathrm{THz}$, but that $t_{y y}$ is close to zero. This means that the $x$-polarized incidence can be converted into $x$ - and $y$-polarized components with the same intensity, and the $y$-polarized incidence can be converted almost completely into the $x$-polarized component at $1 \mathrm{THz}$. Furthermore, the phase difference of $\Delta \delta_{x}$ at $1 \mathrm{THz}$ is approximately $-90^{\circ}$ for an $x$-polarized incidence, which is a necessary condition for LCP. The ellipticities of $E P_{x}$ and $E P_{y}$ at $1 \mathrm{THz}$ are equal to -1 and 0 , corresponding to a LCP and a linear polarization, respectively. To characterize the output polarizations more clearly, we plot the corresponding simulated trajectories of the output polarized electric fields at $1 \mathrm{THz}$ in Fig. 4(a). The arrows indicate the moving directions of the polarized electric field vectors. Obviously, $x$ - and $y$ polarized incidences can be converted into LCP and linear $x$-polarization at $1 \mathrm{THz}$, respectively.
A sample of the ADMM is fabricated through standard electron beam lithography, as described in detail in Ref. [34], for experiments. The MML has a total area of $15 \times 15 \mathrm{~mm}^{2}$ etched on the surface of a 1-mm thick silicon wafer. The scanning electron microscopy image of the sample is shown in the inset of Fig. 5, and the actual measured structural parameters are $a_{1}=81 \mu \mathrm{m}, b_{1}=53 \mu \mathrm{m}$, $a_{2}=69 \mu \mathrm{m}, b_{2}=48 \mu \mathrm{m}, h=191 \mu \mathrm{m}, H=795 \mu \mathrm{m}$, $P=150 \mu \mathrm{m}, \theta_{1}=60^{\circ}$, and $\theta_{2}=30^{\circ}$. A THz time-domain spectroscopy system [34] is used to characterize the polarization conversion performance of the sample, as schematically shown in Fig. 5. Our transmitter and detector are both horizontally polarized in the experiment, and we assume that the horizontal (vertical) direction corresponds to the $x(y)$ direction. $\mathrm{P} 1-\mathrm{P} 4$ are wire-grid linear polarizers, where the polarization conversion processes for a lineally polarized wave follows the Malus law. P2 and P3 are placed at either $0^{\circ}$ or $90^{\circ}$ with respect to the horizontal direction to get an $x$ - or $y$-polarized component, respectively; the former is for the wave incident onto the sample and the latter is for the wave transmitted through the sample. P1 and $\mathrm{P} 4$ are placed at $45^{\circ}$ to ensure an equal polarization projection into the $x$ and $y$ directions; the former is for the required linearly polarized incident wave and the latter is for the measurement of the crosspolarization conversion. The $\mathrm{THz}$ signal is measured in the time domain, and a Fourier transform is performed to convert it into the frequency domain. The material properties can thus be extracted by comparing the spectra of the $\mathrm{THz}$ beams going through the sample and through an air reference. Experimental results are presented in Figs. 3(d)-3(f) and 4(b), which show that the $x$ - and
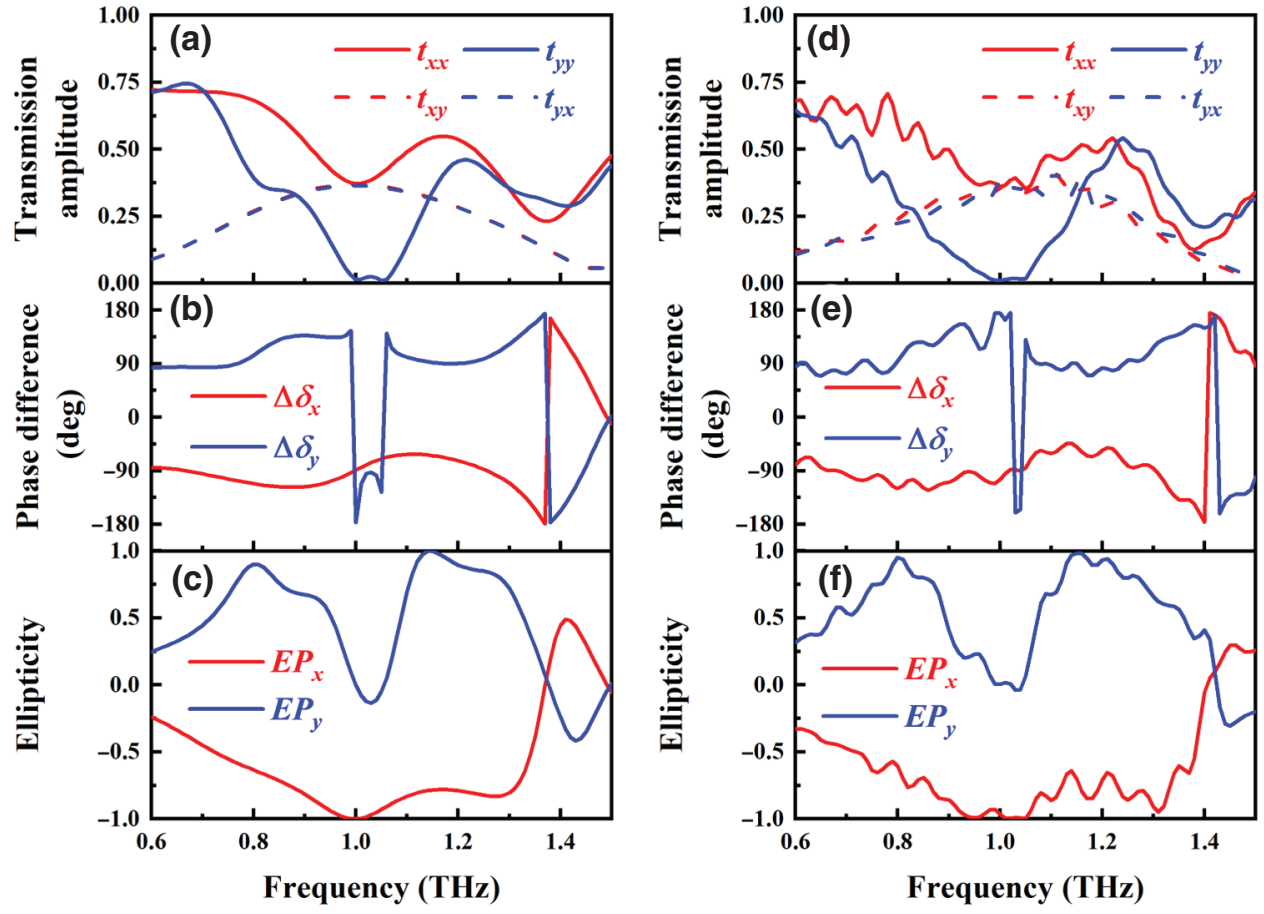

FIG. 3. Simulated (a)-(c) and experimental (d)-(f) results of the ADMM for (a) and (d) the transmission amplitudes, (b) and (e) the phase differences, and (c) and (f) the ellipticities. 

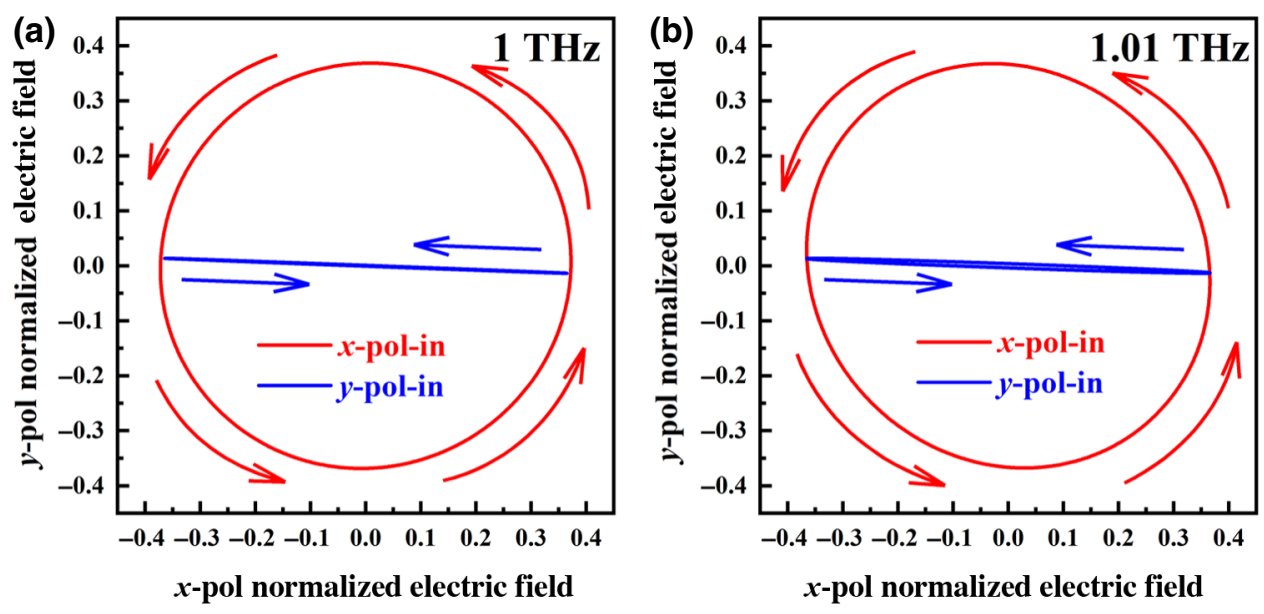

FIG. 4. (a) Simulated trajectories of output polarized electric fields at $1 \mathrm{THz}$. (b) Experimental trajectories of output polarized electric fields at $1.01 \mathrm{THz}$. $y$-polarized incidences are converted into LCP and linear $x$ polarization at a frequency of $1.01 \mathrm{THz}$, respectively. The agreement between experiment and simulation is excellent. The minor differences are mainly caused by the dimension discrepancies of the fabricated sample. A 3D model with the actual structural parameters of the sample is simulated to verify the difference; the corresponding results are shown in Fig. S2 within the Supplemental Material [43].

Polarization plays a vital role in a wide range of fields, including chiral responses in chemical and biological substances [47], polarization-division-multiplexing in optical communications [48], and spin-dependent light-matter interactions [49]. In such applications, a linear-to-circular polarization conversion is often required. Our proposed design fulfills not only this requirement, but also performs an extra linear-to-linear polarization conversion; thus two functions are combined into a single device.

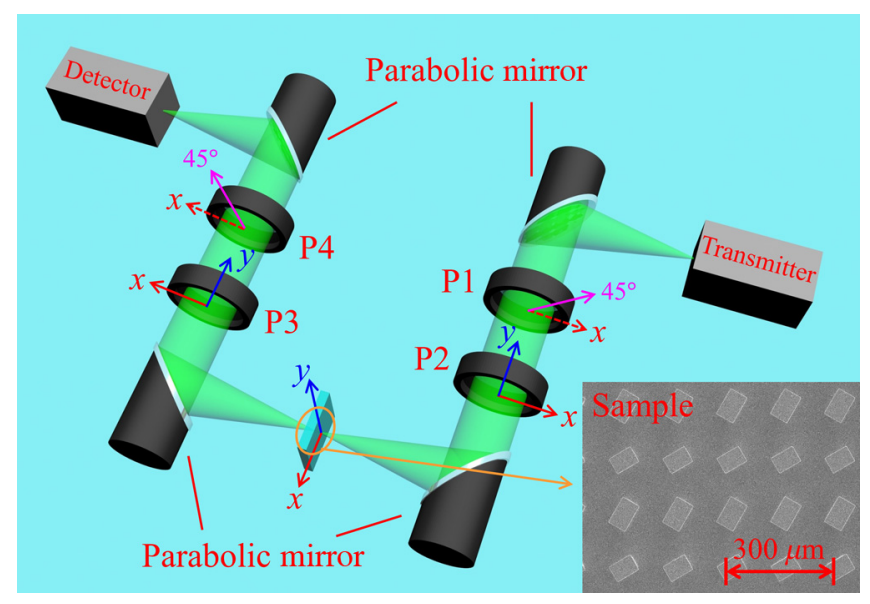

FIG. 5. Schematic of the experimental setup for polarization conversion characterization. Our transmitter and detector are both $x$ polarized. $\mathrm{P} 2$ and $\mathrm{P} 3$ are placed at either $0^{\circ}$ or $90^{\circ}$ with respect to the $x$ direction to get an $x$ - or $y$-polarized wave, respectively. $\mathrm{P} 1$ and $\mathrm{P} 4$ are placed at $45^{\circ}$ to ensure an equal polarization projection into the $x$ and $y$ directions.
More importantly, aside from the dual-functional device itself and the advantage of being compatible with current silicon technology, the design strategy of DPUC has a wider applicability. Metamaterials are widely utilized to design different kinds of functional devices and are typically SSUC-based. However, due to the limitations of the single subwavelength structure, such metamaterials generally have few degrees of design freedom and cannot realize more complex functions. Our DPUC design can be extended to MSUCs, which enables higher degrees of design freedom for functions that cannot be achieved with SSUCs.

\section{CONCLUSION}

Here, we report a design strategy of a DPUC-based ADMM that can perform two different linear-to-circular and linear-to-cross-linear polarization conversions at the same operation frequency. As an example, an ADMM designed to operate at $1 \mathrm{THz}$ can convert $x$ - and $y$ polarized $\mathrm{THz}$ incidences into LCP and $x$ polarization, respectively. An ADMM sample is fabricated and the desired dual-functional performance is achieved at $1.01 \mathrm{THz}$. The design principle of DPUC has wide applicability for functional metamaterial device designs and can be extended to more complex scenarios. The reported dual-functional polarization converter and the associated design strategy will be of significance in the development of versatile $\mathrm{THz}$ devices.

\section{ACKNOWLEDGMENTS}

This work is financially supported by the National Key Research and Development Program of China (Grant No. 2017YFA0701004), the National Natural Science Foundation of China (Grants No. 61875150, No. 61935015, No. 61605143, No. 61735012, No. 61722509, and No. 61871212), the Tianjin Municipal Fund for Distinguished Young Scholars (Grant No. 18JCJQJC45600), and King Abdullah University of Science and Technology (KAUST) 
Office of Sponsored Research (OSR) (Grant No. OSR2016-CRG5-2950).

[1] L. S. Bilbro, R. V. Aguilar, G. Logvenov, O. Pelleg, I. Božović, and N. P. Armitage, Temporal correlations of superconductivity above the transition temperature in $\mathrm{La}_{2-x} \mathrm{Sr}_{x} \mathrm{CuO}_{4}$ probed by terahertz spectroscopy, Nat. Phys. 7, 298 (2011).

[2] C. Jansen, S. Wietzke, O. Peters, M. Scheller, N. Vieweg, M. Salhi, N. Krumbholz, C. Jördens, T. Hochrein, and M. Koch, Terahertz imaging: Applications and perspectives, Appl. Opt. 49, E48 (2010).

[3] T. Nagatsuma, G. Ducournau, and C. C. Renaud, Advances in terahertz communications accelerated by photonics, Nat. Photonics 10, 371 (2016).

[4] X. Shen, Y. Yang, Y. Zang, J. Gu, J. Han, W. Zhang, and T. J. Cui, Triple-band terahertz metamaterial absorber: Design, experiment, and physical interpretation, Appl. Phys. Lett. 101, 154102 (2012).

[5] X. Liu, K. Fan, I. V. Shadrivov, and W. J. Padilla, Experimental realization of a terahertz all-dielectric metasurface absorber, Opt. Express 25, 191 (2017).

[6] M. Wu, X. Zhao, J. Zhang, J. Schalch, G. Duan, K. Cremin, R. D. Averitt, and X. Zhang, A three-dimensional all-metal terahertz metamaterial perfect absorber, Appl. Phys. Lett. 111, 051101 (2017).

[7] I. H. Libon, S. Baumgärtner, M. Hempel, N. E. Hecker, J. Feldmann, M. Koch, and P. Dawson, An optically controllable terahertz filter, Appl. Phys. Lett. 76, 2821 (2000).

[8] S. Pelling, R. Davis, L. Kulik, A. Tzalenchuk, S. Kubatkin, T. Ueda, S. Komiyama, and V. N. Antonov, Point contact readout for a quantum dot terahertz sensor, Appl. Phys. Lett. 93, 073501 (2008).

[9] H. T. Chen, W. J. Padilla, M. J. Cich, A. K. Azad, R. D. Averitt, and A. J. Taylor, A metamaterial solid-state terahertz phase modulator, Nat. Photonics 3, 148 (2009).

[10] M. Rahm, J. S. Li, and W. J. Padilla, THz wave modulators: A brief review on different modulation techniques, J. Infrared, Millimeter, Terahertz Waves 34, 1 (2013).

[11] L. Ren, C. L. Pint, L. G. Booshehri, W. D. Rice, X. Wang, D. J. Hilton, K. Takeya, I. Kawayama, M. Tonouchi, R. H. Hauge, and J. Kono, Carbon nanotube terahertz polarizer, Nano Lett. 9, 2610 (2009).

[12] W. S. L. Lee, S. Nirantar, D. Headland, M. Bhaskaran, S. Sriram, C. Fumeaux, and W. Withayachumnankul, Broadband terahertz circular-polarization beam splitter, Adv. Opt. Mater. 6, 1700852 (2018).

[13] L. Zhang, C. R. Donaldson, and W. He, Design and measurement of a polarization convertor based on a truncated circular waveguide, J. Phys. D. Appl. Phys. 45, 345103 (2012).

[14] J.-B. Masson and G. Gallot, Terahertz achromatic quarterwave plate, Opt. Lett. 31, 265 (2006).

[15] K. Wiesauer and C. Jördens, Recent advances in birefringence studies at $\mathrm{THz}$ frequencies, J. Infrared, Millimeter, Terahertz Waves 34, 663 (2013).

[16] A. K. Kaveev, G. I. Kropotov, E. V. Tsygankova, I. A. Tzibizov, S. D. Ganichev, S. N. Danilov, P. Olbrich, C.
Zoth, E. G. Kaveeva, A. I. Zhdanov, A. A. Ivanov, R. Z. Deyanov, and B. Redlich, Terahertz polarization conversion with quartz waveplate sets, Appl. Opt. 52, B60 (2013).

[17] S. Liu and T. J. Cui, Concepts, working principles, and applications of coding and programmable metamaterials, Adv. Opt. Mater. 5, 1700624 (2017).

[18] D. Headland, E. Carrasco, S. Nirantar, W. Withayachumnankul, P. Gutruf, J. Schwarz, D. Abbott, M. Bhaskaran, S. Sriram, J. Perruisseau-Carrier, and C. Fumeaux, Dielectric resonator reflectarray as high-efficiency nonuniform terahertz metasurface, ACS Photonics 3, 1019 (2016).

[19] S. B. Glybovski, S. A. Tretyakov, P. A. Belov, Y. S. Kivshar, and C. R. Simovski, Metasurfaces: From microwaves to visible, Phys. Rep. 634, 1 (2016).

[20] J. W. Stewart, G. M. Akselrod, D. R. Smith, and M. H. Mikkelsen, Toward multispectral imaging with colloidal metasurface pixels, Adv. Mater. 29, 1602971 (2017).

[21] I. Al-Naib and W. Withayachumnankul, Recent progress in terahertz metasurfaces, J. Infrared, Millimeter, Terahertz Waves 38, 1067 (2017).

[22] N. K. Grady, J. E. Heyes, D. R. Chowdhury, Y. Zeng, M. T. Reiten, A. K. Azad, A. J. Taylor, D. A. R. Dalvit, and H. T. Chen, Terahertz metamaterials for linear polarization conversion and anomalous refraction, Science 340, 1304 (2013).

[23] Y. Yang, W. Wang, P. Moitra, I. I. Kravchenko, D. P. Briggs, and J. Valentine, Dielectric meta-reflectarray for broadband linear polarization conversion and optical vortex generation, Nano Lett. 14, 1394 (2014).

[24] M. Chen, F. Fan, S. T. Xu, and S. J. Chang, Artificial high birefringence in all-dielectric gradient grating for broadband terahertz waves, Sci. Rep. 6, 38562 (2016).

[25] Z. Liu, Z. Li, Z. Liu, H. Cheng, W. Liu, C. Tang, C. Gu, J. Li, H. T. Chen, S. Chen, and J. Tian, Single-layer plasmonic metasurface half-wave plates with wavelengthindependent polarization conversion angle, ACS Photonics 4, 2061 (2017).

[26] X. Ke, H. Zhu, J. Li, L. Chen, and X. Li, Double-stacked hyperbolic metamaterial waveguide arrays for efficient and broadband terahertz quarter-wave plates, Sci. Rep. 7, 574 (2017).

[27] J. M. Woo, S. Hussain, and J. H. Jang, A terahertz inline polarization converter based on through-via connected double layer slot structures, Sci. Rep. 7, 42952 (2017).

[28] W. S. L. Lee, R. T. Ako, M. X. Low, M. Bhaskaran, S. Sriram, C. Fumeaux, and W. Withayachumnankul, Dielectricresonator metasurfaces for broadband terahertz quarter- and half-wave mirrors, Opt. Express 26, 14392 (2018).

[29] A. C. Strikwerda, K. Fan, H. Tao, D. V. Pilon, X. Zhang, and R. D. Averitt, Comparison of birefringent electric splitring resonator and meanderline structures as quarter-wave plates at terahertz frequencies, Opt. Express 17, 136 (2009).

[30] Y. Z. Cheng, W. Withayachumnankul, A. Upadhyay, D. Headland, Y. Nie, R. Z. Gong, M. Bhaskaran, S. Sriram, and D. Abbott, Ultrabroadband reflective polarization convertor for terahertz waves, Appl. Phys. Lett. 105, 181111 (2014).

[31] W. Liu, S. Chen, Z. Li, H. Cheng, P. Yu, J. Li, and J. Tian, Realization of broadband cross-polarization conversion in 
transmission mode in the terahertz region using a singlelayer metasurface, Opt. Lett. 40, 3185 (2015).

[32] Y. Jiang, L. Wang, J. Wang, C. N. Akwuruoha, and W. Cao, Ultra-wideband high-efficiency reflective linear-to-circular polarization converter based on metasurface at terahertz frequencies, Opt. Express 25, 27616 (2017).

[33] J. Zi, Q. Xu, Q. Wang, C. Tian, Y. Li, X. Zhang, J. Han, and W. Zhang, Terahertz polarization converter based on all-dielectric high birefringence metamaterial with elliptical air holes, Opt. Commun. 416, 130 (2018).

[34] J. Zi, Q. Xu, Q. Wang, C. Tian, Y. Li, X. Zhang, J. Han, and W. Zhang, Antireflection-assisted all-dielectric terahertz metamaterial polarization converter, Appl. Phys. Lett. 113, 101104 (2018).

[35] A. Kumar and A. Ghatak, Polarization of Light with Applications in Optical Fibers (SPIE Press, Bellingham, WA, USA, 2011).

[36] X. Fu and T. J. Cui, Recent progress on metamaterials: From effective medium model to real-time information processing system, Prog. Quantum Electron. 67, 100223 (2019).

[37] Q. Yang, X. Chen, Q. Xu, C. Tian, Y. Xu, L. Cong, X. Zhang, Y. Li, C. Zhang, X. Zhang, J. Han, and W. Zhang, Broadband terahertz rotator with an all-dielectric metasurface, Photonics Res. 6, 1056 (2018).

[38] N. Yu, F. Aieta, P. Genevet, M. A. Kats, Z. Gaburro, and F. Capasso, A broadband, background-free quarter-wave plate based on plasmonic metasurfaces, Nano Lett. 12, 6328 (2012).

[39] F. Ding, Z. Wang, S. He, V. M. Shalaev, and A. V. Kildishev, Broadband high-efficiency half-wave plate: A supercell-based plasmonic metasurface approach, ACS Nano 9, 4111 (2015).

[40] Z. L. Deng, J. Deng, X. Zhuang, S. Wang, K. Li, Y. Wang, Y. Chi, X. Ye, J. Xu, G. P. Wang, R. Zhao, X. Wang, Y. Cao, X. Cheng, G. Li, and X. Li, Diatomic metasurface for vectorial holography, Nano Lett. 18, 2885 (2018).
[41] S. Wang, F. Li, J. Deng, X. Ye, Z.-L. Deng, Y. Cao, B.-O. Guan, G. Li, and X. Li, Diatomic metasurface based broadband J-plate for arbitrary spin-to-orbital conversion, J. Phys. D. Appl. Phys. 52, 324002 (2019).

[42] F. Zhang, M. Pu, X. Li, P. Gao, X. Ma, J. Luo, H. Yu, and X. Luo, All-dielectric metasurfaces for simultaneous giant circular asymmetric transmission and wavefront shaping based on asymmetric photonic spin-orbit interactions, Adv. Funct. Mater. 27, 1704295 (2017).

[43] See the Supplemental Material at http://link.aps.org/supple mental/10.1103/PhysRevApplied.13.034042 for the determination of the parameters of the dual pillars used in the design, further analysis of the working principles of the dual-pillar structure, and a comparison between experiment and simulation for the fabricated sample.

[44] A. Arbabi, Y. Horie, M. Bagheri, and A. Faraon, Dielectric metasurfaces for complete control of phase and polarization with subwavelength spatial resolution and high transmission, Nat. Nanotechnol. 10, 937 (2015).

[45] E. Arbabi, A. Arbabi, S. M. Kamali, Y. Horie, and A. Faraon, Multiwavelength metasurfaces through spatial multiplexing, Sci. Rep. 6, 32803 (2016).

[46] A. Arbabi, Y. Horie, A. J. Ball, M. Bagheri, and A. Faraon, Subwavelength-thick lenses with high numerical apertures and large efficiency based on high-contrast transmitarrays, Nat. Commun. 6, 7069 (2015).

[47] Z. Wang, F. Cheng, T. Winsor, and Y. Liu, Optical chiral metamaterials: A review of the fundamentals, fabrication methods and applications, Nanotechnology 27, 412001 (2016).

[48] J. Wang, S. He, and D. Dai, On-chip silicon 8-channel hybrid (de)multiplexer enabling simultaneous mode- and polarization-division-multiplexing, Laser Photonics Rev. 8, L18 (2014).

[49] S. Xiao, J. Wang, F. Liu, S. Zhang, X. Yin, and J. Li, Spindependent optics with metasurfaces, Nanophotonics 6, 215 (2017). 